\title{
Pautas metodológicas en el abordaje de la oralidad en la escuela primaria costarricense
}

\author{
Methodological guidelines in dealing with orality in \\ Costa Rican elementary school
}

\author{
Jéssica Araya Ramírez \\ Profesora Escuela de Formación Docente, Facultad de Educación \\ Universidad de Costa Rica \\ Costa Rica \\ jesaraya30@yahoo.es
}

Recibido: 26-VIII-2011 • Aceptado 20-XI-2011 • Corregido 15-XII-2011

\begin{abstract}
Resumen: En el presente artículo se describen una serie de pautas metodológicas para el aprendizaje graduado y sistematizado de la expresión oral en escolares, las cuales se plantean a partir de los resultados obtenidos por Araya (2011) en el desarrollo de la investigación denominada "La competencia en la expresión oral de escolares en Costa Rica. El componente léxico". En esta se describe el vocabulario en cuanto a diversidad y precisión en relación con la producción de textos orales narrativos y explicativos en el plano oral, en el cual se presentó un comportamiento poco evolutivo sobre el desarrollo del léxico.
\end{abstract}

Palabras clave: Metodología, estrategias, explicativos, narrativos, textos, oral, didáctica educativo.

\section{Introducción}

La expresión oral en la escuela primaria es una habilidad lingüística que plantea la comunicación coherente, concisa y adecuada en diversas situaciones del habla espontánea y formal, por lo que la combinación de recursos lingüísticos y no lingüísticos es necesaria para producir e interpretar mensajes con diferentes intenciones comunicativas. Para lograr lo anterior, es indispensable la planificación sistematizada y graduada en el aula de situaciones discursivas espontáneas y, posteriormente, más formales, en las que las destrezas de la oralidad puedan ser adquiridas y ejercitadas en forma sistemática a través de los grados escolares.

En este sentido, la tendencia por abordar en su mayoría los procesos de 


\begin{abstract}
This article describes a number of methodological guidelines for systematic learning of the graduate and speaking in schools, which arise from the results obtained by Araya (2011), in the development of research called "Competition oral expression of students in Costa Rica. The token ", the author of this publication, in which the token was analyzed in terms of diversity and precision of vocabulary in relation to the production of oral narrative and explanatory texts at the mouth, where he presented a short performance evolution on vocabulary development.
\end{abstract}

Keywords: Methodology, strategies, explanatory, narrative, texts, oral, didactics lectura y escritura en las aulas no ha permitido la sistematización de los procesos de aprendizaje de las competencias orales, ya que los docentes asumen que los niños al ingresar a la escuela ya saben hablar y, por lo tanto, no es necesaria una mediación pedagógica que norme y permita acrecentar las habilidades lingüísticas.

Debido a esa percepción, es poca la intervención didáctica que se da en el aula y se deja de lado el negociar significados, construir conocimientos, explicar hechos y argumentar opiniones de una forma planeada y graduada.

Específicamente, las investigaciones realizadas sobre la expresión oral en nuestro país se relacionan con el desarrollo del léxico, las cuales han permitido fundamentar la importancia de este componente lingüístico-textual en el abordaje de los procesos de aprendizaje de la oralidad. Entre estos estudios se citan el propuesto por Murillo (1999) sobre los registros de disponibilidad léxica, que orientan la planificación de la enseñanza de la lengua por grados o niveles educativos, pues proporcionan el vocabulario usual del habla en situaciones específicas.

Por otro lado, se han desarrollado investigaciones en las que se han estructurado propuestas de léxico básico y meta. En este sentido los estudios de Murillo y Sánchez (2002), Murillo (2003) y Rojas (2002 y 2005) permiten visualizar una descripción detallada del registro del vocabulario básico utilizado por los escolares costarricenses y, a partir de ese repertorio, proyectan el léxico meta de la población estudiantil. Igualmente estos estudios han permitido elaborar propuestas metodológicas dirigidas a la adquisición y desarrollo del léxico, tal es el caso de los estudios de Murillo (sin publicar), Rojas (2006 b) y Rojas et ál. (2008), propuestas que se convierten en herramientas que permiten fortalecer el léxico de los escolares en forma progresiva y sistemática. 
Aunado a ello, como parte del comportamiento del léxico en escolares, es importante identificar y analizar la influencia de patrones y elementos culturales en la producción de palabras, de ahí que las investigaciones realizadas por Murillo y Sánchez (2004 y 2005) permiten determinar que el vocabulario de los niños escolares es complejo y muestra una cantidad diversa de elementos culturales que reflejan la percepción de su entorno. Lo anterior debe ser considerado por los docentes en la planificación de la enseñanza del léxico.

Recientemente, Araya (2011) desarrolla un estudio para valorar el comportamiento del componente léxico (diversidad y precisión léxica) en textos orales narrativos y explicativos. En este sentido, se consideró como antecedente los resultados obtenidos por Barboza y Delgado (2008) sobre la producción escrita que realizan los escolares en Costa Rica, donde se demuestra que los niños al escribir producen mayoritariamente estas tipologías textuales. En correspondencia con esto, la investigación desarrollada por Araya (2011) contempló estos textos para analizar el desarrollo oral de los escolares en cuanto al componente léxico.

Por otro lado, con el propósito de enmarcar las pautas metodológicas que se proponen en este artículo con el objetivo de fortalecer la oralidad en los escolares, se describen primeramente los objetivos de la expresión oral en la educación primaria y su contextualización en el sistema educativo costarricense; asimismo, los contenidos lingüísticos y no lingüísticos que los escolares deben adquirir y desarrollar para expresarse en forma oral de manera adecuada. Posteriormente, se presenta una síntesis de los resultados obtenidos de la investigación realizada por Araya (2011), los cuales son los hallazgos que dan sustento a las pautas metodológicas que se esbozan al final de la presente publicación.

\section{Fundamentos conceptuales}

\subsection{Objetivos de la expresión oral en la educación primaria}

Tal como señala Palou et ál. (2005), la enseñanza de la lengua oral en las escuelas persigue dos motivos: el papel mediador que desempeña en los procesos de aprendizaje y la consecución de los objetivos relacionados con la formación de futuros ciudadanos.

En consecuencia, dichos propósitos a su vez persiguen la adquisición de habilidades y destrezas necesarias para que el individuo sea competente en el uso y la combinación de recursos lingüísticos y no lingüísticos que le permitan producir e interpretar mensajes, según la intencionalidad, atendiendo las pautas propias de cada intercambio comunicativo.

Lo anterior se sustenta con el pensamiento de Vigotsky (1934), citado por Azagra et ál. (1998), en cuanto a considerar el lenguaje como un instrumento necesario para organizar el pensamiento, razonar, desarrollar el conocimiento y planificar acciones, así como un instrumento cultural, político, social y económico. Un desarrollo que integre el pensamiento y la cultura se debe no solo a la necesidad que tiene el niño de aprender la lengua, sino también a la urgencia de usarla para poder participar en las situaciones de la vida diaria. Teniendo en cuenta lo anterior, la escuela se convierte en un espacio social imprescindible para gestar la conversación y el diálogo entre docentes y alumnos.

El aprendizaje de la expresión oral requiere un planteamiento de objetivos concretos sobre el qué, cómo y para qué enseñar la lengua oral, así como el diseño de una evaluación formativa sustentada en la reflexión sobre el dominio de la oralidad. En este sentido, se deberá cuestionar cómo es el espacio de participación que se le otorga a los sujetos, el tipo de intervenciones que hacen los estudiantes, cuáles son las 
competencias orales que se persiguen en los grados escolares y, por último, cómo establecer criterios que permitan a los estudiantes mejorar y progresar. Tales tareas permitirán ahondar más en el desarrollo evolutivo del escolar en cuanto a la expresión oral de tal forma que puedan establecerse estadios en los que se pueda identificar el nivel de dominio de habilidades, destrezas y conocimientos indispensables en la producción de la oralidad.

Por su parte Reyzábal (1993) presenta detalladamente los objetivos de la oralidad, lo que permite establecer una enseñanza gradual de la misma:

- Comprender mensajes orales, relacionándolos con las propias ideas y experiencias, interpretándolas y valorándolas críticamente y aplicándolos a nuevas situaciones de aprendizaje.

- Utilizar la lengua oral para intercambiar ideas, experiencias y sentimientos, adoptando una actitud respetuosa ante el aporte de los otros.

- Expresarse oralmente teniendo en cuenta las características de las situaciones de comunicación y respetando las formas básicas de la lengua oral (progresiva corrección en la pronunciación, en las estructuras morfosintácticas, en las estructurales textuales, en el uso del léxico, entre otros).

- Utilizar diferentes recursos expresivos lingüísticos, no lingüísticos y paralingüísticos en los discursos orales.

- Utilizar el lenguaje oral como instrumento de aprendizaje para planificar una actividad.

- Reconocer la existencia de los distintos usos y registros en las producciones lingüísticas propias y ajenas.

- Comprender y producir textos orales con características literarias en los que se reconozca y disfrute sus recursos expresivos y riqueza estético-lingüística.

- Valorar la literatura de tradición oral como patrimonio cultural enriquecedor (p. 134).
Dichos objetivos promueven las competencias orales en general, ya que se parte de la comprensión de mensajes orales, lo que requiere de habilidades en el área de la escucha para poder identificar detalles del mensaje y de esta manera producir argumentos, comentarios, entre otros. Igualmente a través de este planteamiento de objetivos, se pretende que el sujeto pueda desarrollar discursos según la situación comunicativa y para ello debe integrar los conocimientos lingüísticos, no lingüísticos y paralingüísticos del discurso oral.

Los objetivos anteriores no solo determinan el qué, para qué y el cómo de la oralidad, sino que también permiten visualizar que esta, a pesar de ser espontánea y natural, requiere de un programa sistemático, graduado y centrado en competencias, que permita retroalimentar géneros discursivos más formales como la conferencia, el debate, entre otros.

De esta manera, los objetivos se formularían a partir de capacidades y no de contenidos. Los ejes temáticos deben abordarse desde los conocimientos previos de los estudiantes y la función del docente es la de descubrir y explorar dichos saberes. Además, en cuanto a la evaluación, esta deberá centrarse en el dominio de capacidades.

La visión de la enseñanza de la lengua se ha ampliado y existe preocupación por las áreas que se han descuidado. Los avances tecnológicos, la era de la información y la especialización del conocimiento exigen al individuo un nivel de comunicación oral tan competente como la expresión escrita. Las carencias en la comunicación oral no solo limitan la interacción social, sino también obstaculizan las potencialidades y las capacidades de la persona e inciden en su desarrollo académico y profesional.

Lomas (1999) señala la importancia de analizar "los usos lingüísticos en las aulas viéndolos no sólo como medios de transmisión de conocimientos sino como herramientas de producción del conocimiento. Los nuevos enfoques educativos 
buscan replantear la enseñanza para que deje de ser una mera transmisión de conocimientos" (p. 304)

Dentro del enfoque comunicativo es fundamental cuestionar si el contexto del aula brinda oportunidades para que los estudiantes practiquen las normas de interacción en la clase necesarias para la enseñanza de la lengua oral.

Además, al basarse en una programación sistematizada y graduada de habilidades comunicativas, el enfoque comunicativo implica que la enseñanza de la lengua oral deba incorporar en una primera etapa un proceso de aprendizaje que le permita al estudiante construir tipos de textos orales en los que incorpore sus respectivas características para que, posteriormente, pueda trabajar con géneros discursivos más formales. Al respecto, Murillo (2003) considera que el enfoque comunicativo es el que toma como punto de partida el capital lingüístico de los educandos $\mathrm{y}$, a partir de este, orienta los procesos de intervención pedagógica hacia una competencia meta.

En este caso, la escuela tiene la responsabilidad de planificar situaciones de aprendizaje que involucren un crecimiento de las competencias orales, ya que muchas veces el entorno social o familiar no genera las posibilidades para practicarlas. Por lo tanto, los objetivos que corresponden a la intervención didáctica de la expresión oral deben partir del enfoque comunicativo para que sea perfeccionado dentro de un trabajo continuo, riguroso y sistemático.

\subsection{Objetivos de la expresión oral en la educación primaria costarricense}

En la actualidad no se cuenta en el país con investigaciones que brinden una sistematización del aprendizaje de la lengua oral, el único documento oficial que hace alusión a los objetivos de la oralidad en la escuela costarricense es la versión actual del programa de estudio de español del
Ministerio de Educación Pública (2004), en el cual se visualiza la promoción de las cuatro habilidades lingüísticas: escritura, escucha, lectura y la expresión oral. Esta última se describe como la capacidad comunicativa de vivencias e intereses de los niños y el intercambio de experiencias en juegos y actividades. Para lograrlo hay que practicar la dicción y una utilización adecuada de la voz, así como el enriquecimiento léxico.

El programa de español incorpora los siguientes objetivos generales de expresión oral para primero y segundo ciclos:

Tabla 1

Objetivos de la expresión oral en la educación primaria costarricense

\begin{tabular}{|c|c|}
\hline I Ciclo & II Ciclo \\
\hline $\begin{array}{l}\text { - Comprender los con- } \\
\text { ceptos relativos a los } \\
\text { elementos del proceso } \\
\text { comunicativo. } \\
\text { - Relatar experiencias } \\
\text { de personas, cuentos, } \\
\text { fábulas y leyendas. } \\
\text { - Producir descripcio- } \\
\text { nes orales. } \\
\text { - Ejercitar las des- } \\
\text { trezas básicas en la } \\
\text { expresión oral de los } \\
\text { alumnos. }\end{array}$ & $\begin{array}{l}\text { - Aplicar los conceptos } \\
\text { relativos al proceso } \\
\text { comunicativo. } \\
\text { - Enriquecer el léxico } \\
\text { junto con el significado de } \\
\text { las palabras. } \\
\text { - Reconocer oraciones como } \\
\text { unidades de discurso. } \\
\text { - Producir descripciones } \\
\text { orales según las normas } \\
\text { idiomáticas. } \\
\text { - Relatar experiencias de } \\
\text { personas, cuentos, fábulas } \\
\text { y leyendas. }\end{array}$ \\
\hline
\end{tabular}

Fuente: Ministerio de Educación Pública Programa de estudio de español (2004, cfr. p. 26-27)

Esta distribución muestra un sesgo hacia los textos orales descriptivos y narrativos, dejando de lado otras tipologías textuales. Además, la interpretación es ambigua en algunos casos, pues los objetivos generales no se encuentran bien delimitados y el planteamiento de logros del segundo ciclo podría ser desarrollado desde el primer ciclo. Además, no se puntualiza una evaluación con criterios específicos, necesarios para modular la programación de la lengua oral a lo largo de la escolaridad. 
En relación con las debilidades anteriores, Murillo (2008) estableció una sistematización de los contenidos de la expresión oral en el contexto escolar costarricense, los cuales se encuentran organizados de primero a sexto grado. La investigadora los denominó logros mínimos para cada uno de los niveles escolares (primero a sexto grado) y responden, respecto de la expresión oral, a los siguientes objetivos generales:

Primer ciclo:

1. Comunicar oralmente ideas $\mathrm{y}$ experiencias, atendiendo las reglas propias de la interacción lingüística.

2. Producir textos narrativos, descriptivos, explicativos, expositivos y conversacionales, según los sistemas comunicativos en que participen los escolares.

3. Comprender e interpretar diferentes textos orales asumiendo una actitud crítica.

Segundo ciclo:

1. Producir textos narrativos, descriptivos, explicativos, expositivos y conversacionales, según los sistemas comunicativos en que participen los escolares.

2. Dominio de las microhabilidades: interpretación, reconocimiento, selección, inferencia y retención en la escucha de los diferentes tipos de texto oral.

De acuerdo con los anteriores objetivos se presentan a continuación los logros mínimos:

Tabla 2

Logros mínimos en el aprendizaje de la expresión oral

\begin{tabular}{|c|c|c|c|c|c|c|}
\hline Logros mínimos & I & II & III & IV & $\mathrm{V}$ & VI \\
\hline $\begin{array}{l}\text { 1. Utiliza adecuadamente elementos lingüísticos para } \\
\text { expresar ideas. }\end{array}$ & $\mathrm{X}$ & $\mathrm{X}$ & $\mathrm{X}$ & $\mathrm{X}$ & $\mathrm{X}$ & $\mathrm{X}$ \\
\hline $\begin{array}{l}\text { 2. Produce textos argumentativos, narrativos, descriptivos } \\
\text { y explicativos. }\end{array}$ & $\mathrm{X}$ & $\mathrm{X}$ & $\mathrm{X}$ & $\mathrm{X}$ & $\mathrm{X}$ & $\mathrm{X}$ \\
\hline 3. Reproduce textos orales. & $\mathrm{X}$ & $\mathrm{X}$ & $\mathrm{X}$ & $\mathrm{X}$ & $\mathrm{X}$ & $\mathrm{X}$ \\
\hline 4. Produce textos orales de manera estructurada. & $\mathrm{X}$ & $\mathrm{X}$ & $\mathrm{X}$ & $\mathrm{X}$ & $\mathrm{X}$ & $\mathrm{X}$ \\
\hline 5. Usa elementos paralingüísticos. & $\mathrm{X}$ & $\mathrm{X}$ & $\mathrm{X}$ & $\mathrm{X}$ & $\mathrm{X}$ & $\mathrm{X}$ \\
\hline 6. Practica diferentes estrategias de expresión oral. & & $\mathrm{X}$ & $\mathrm{X}$ & $\mathrm{X}$ & $\mathrm{X}$ & $\mathrm{X}$ \\
\hline 7. Utiliza el lenguaje con intenciones determinadas. & & & $\mathrm{X}$ & $\mathrm{X}$ & $\mathrm{X}$ & $\mathrm{X}$ \\
\hline 8. Utiliza soportes escritos para preparar sus intervenciones orales. & & & $\mathrm{X}$ & $\mathrm{X}$ & $\mathrm{X}$ & $\mathrm{X}$ \\
\hline 9. Capta el tono de producciones orales. & & & $\mathrm{X}$ & $\mathrm{X}$ & $\mathrm{X}$ & $\mathrm{X}$ \\
\hline $\begin{array}{l}\text { 10. Aprovecha el tiempo para decir todo lo que se considere necesario } \\
\text { en las interacciones comunicativas. }\end{array}$ & & & $\mathrm{X}$ & $\mathrm{X}$ & $\mathrm{X}$ & $\mathrm{X}$ \\
\hline 11. Selecciona temas adecuados para expresarse. & & & & $\mathrm{X}$ & $\mathrm{X}$ & $\mathrm{X}$ \\
\hline 12. Coopera como miembro activo de las interacciones verbales. & & & & $\mathrm{X}$ & $\mathrm{X}$ & $\mathrm{X}$ \\
\hline 13. Identifica intenciones explícitas e implícitas de la comunicación. & & & & $\mathrm{X}$ & $\mathrm{X}$ & $\mathrm{X}$ \\
\hline 14. Comprende las formas de estructurar el discurso. & & & & & $\mathrm{X}$ & $\mathrm{X}$ \\
\hline 15. Adapta el grado de especificaciones de los textos orales. & & & & & & $\mathrm{X}$ \\
\hline 16. Controla la voz. & & & & & & $\mathrm{X}$ \\
\hline 17. Usa códigos no verbales adecuados. & & & & & & $\mathrm{X}$ \\
\hline
\end{tabular}

Fuente: Murillo, 2008 
Esta propuesta demuestra cuán necesario es formular un planteamiento detallado que concrete las metas, los contenidos y las habilidades del código oral que los estudiantes deben desarrollar. En este caso los aspectos sintácticos, morfológicos, léxicos y organizacionales del discurso oral deben sistematizarse en cada uno de los grados escolares para evitar las improvisaciones y fundamentar sobre el cómo desarrollar esta habilidad lingüística en los ambientes educativos.

De ahí la urgente necesidad de que haya una intervención didáctica en la expresión oral y que esta sea replanteada con los fundamentos teóricos necesarios para poder visualizar más claramente cuáles son los principios de enseñanza con los que se puede mejorar la adquisición de dicha habilidad.

En la actualidad el sistema educativo costarricense evalúa la oralidad como un producto final, mediante hojas de cotejo, basándose en los objetivos propuestos de los programas de español emanados por el Ministerio de Educación Pública; sin embargo, la evaluación se realiza como un requisito más, pero no existe un proceso que permita que los niños se apropien e interactúen con las habilidades lingüísticas y no lingüísticas para producir el discurso.

\subsection{Las habilidades y conocimientos discursivos que deben incursionarse gradualmente en el aprendizaje de la expresión oral en la escuela primaria}

La adquisición y desarrollo de la competencia oral es un proceso gradual, el cual se fortalece mediante las experiencias comunicativas que los escolares enfrenten en diversas situaciones. En este sentido, es necesario no solo de un dominio del componente gramatical, sino también de las normas y de la intención de la comunicación.

Se pretende que el dominio de la oralidad le permita al niño una transición de la construcción de textos orales en conversaciones espontáneas para posteriormente generar otro tipo de género, tales como mesas redondas, conferencias, debates, entre otros. Para alcanzar dicho objetivo, se requiere de fundamentos teóricos que permitan visualizar los elementos de la comunicación oral que los escolares deben aprender en forma graduada con el propósito de ser competentes en esta habilidad lingüística. En este caso, Bygate, 1987, citado por Cassany et ál. (1994) ofrece un planteamiento de los elementos que conforman un modelo explicativo que orienta el aprendizaje de los elementos de la oralidad en la escuela primaria. En esta propuesta se identifican una serie de conocimientos y habilidades las cuales de manera integrada permiten una adecuada comunicación oral:

Tabla 3

Modelo explicativo de la oralidad

\begin{tabular}{|c|c|}
\hline Conocimientos & Habilidades \\
\hline $\begin{array}{l}\text { PLANIFICAR } \\
\text { - Conocimiento de } \\
\text { rutinas: información e } \\
\text { interacción } \\
\text { - Conocimiento del } \\
\text { discurso que se va } \\
\text { construyendo }\end{array}$ & $\begin{array}{l}\text { - Planificar el mensaje: } \\
\text { planes de información e } \\
\text { interacción. } \\
\text { - Habilidades de conduc- } \\
\text { ción: conducir el tema } \\
\text { y conceder turnos de la } \\
\text { palabra }\end{array}$ \\
\hline $\begin{array}{l}\text { SELECCIONAR } \\
\text { - Léxico } \\
\text { - Frases } \\
\text { - Recursos } \\
\text { gramaticales }\end{array}$ & $\begin{array}{l}\text { - Habilidades de negocia- } \\
\text { ción de los significados: } \\
\text { grado de explicitación } \\
\text { y evaluación de la } \\
\text { comprensión }\end{array}$ \\
\hline $\begin{array}{l}\text { PRODUCIR } \\
\text { - Mecanismos de } \\
\text { producción } \\
\text { - Reglas gramaticales } \\
\text { y de pronunciación }\end{array}$ & $\begin{array}{l}\text { - Habilidades de produc- } \\
\text { ción: facilitación y } \\
\text { compensación } \\
\text { - Habilidades de } \\
\text { corrección }\end{array}$ \\
\hline
\end{tabular}

Fuente: Bygate,1987, citado por Cassany et ál. (1994:142)

En este modelo se pueden distinguir una serie de competencias, es decir de habilidades y conocimientos, que permiten el hecho comunicativo en el nivel oral. Los 
conocimientos se operacionalizan en tres etapas: la planificación, la selección y la producción y se relacionan con las diversas habilidades que se esperan alcanzar con el dominio de los contenidos o conocimientos. En esta propuesta, los conocimientos mencionados son informaciones que incluyen el dominio del sistema de la lengua (gramática, léxico, morfosintaxis, entre otros) y aspectos relacionados con la cultura. Por otro lado, las habilidades señaladas se refieren a los comportamientos durante los actos de expresión: las habilidades de adaptarse al tema, de adecuar el lenguaje al contexto y la intención comunicativa.

Este modelo de la expresión oral se complementa con la incorporación de la habilidad de autocorrección gramatical considerando las reglas de la gramática y la pronunciación, permitiendo la corrección de los errores en la marcha del discurso, así como con el tono de la voz y la comunicación no verbal (como la postura, inclinación del cuerpo y los gestos)

Asimismo, el esquema explicativo anterior se amplía y se retroalimenta con las características de cada uno de los componentes del discurso oral, señalados por Wittgenstein, citado por Calsamiglia y Tusón (2008), los cuales se convierten en los elementos que deben fundamentar cualquier propuesta curricular para trabajar la oralidad.

En este caso el componente fónico, se refiere a la pronunciación y la prosodia (entonación de los diferentes enunciados interrogativos y exclamativos, marcar el énfasis en una frase, distinguir los ritmos y grupos tonales tales como las pausas de ritmo lento y relajado). Por otro lado, el componente morfosintáctico, que hace alusión a la producción oral planificada, la cual responde a patrones retóricos elaborados dado que la complejidad es más estructurada en el plano sintáctico. Asimismo, en la producción oral más espontánea se presentan frecuentes discordancias, repeticiones, uso de "muletillas", utilización de la yuxtaposición y la coordinación para relacionar oraciones e incluso alteraciones sintácticas que se van resolviendo en el momento de la conversación. Las subordinaciones son menos utilizadas. En el caso del componente léxico, en relación con el código oral, la variación sirve para marcar el tono de la interacción y las finalidades y motivos que se persiguen. También puede indicar características socioculturales y ser marcador de pertenencia a un grupo. Igualmente, este componente se refiere al grado de densidad y precisión léxica, presentes como características del discurso oral (considerando el grado de espontaneidad de la interacción comunicativa).

Por último, se describe el componente organizacional y discursivo, el cual se refiere a la estructura del discurso: inicio, progresión informativa y finalización del mismo. Dentro de la organización del texto se observan marcas de coherencia tales como marcadores discursivos, conectores y marcas de interacción.

En este sentido, es evidente que cada uno de los componentes de la oralidad, los cuales son unidades de análisis, son los elementos que deben canalizarse desde que los escolares van adquiriendo el código oral y que, a la vez, la mediación pedagógica debe explotar a través de la interacción en el aula.

Tanto los objetivos como los contenidos del programa de estudio de español, hacen hincapié en cada uno de esos elementos; por lo tanto, el profesorado debe tener un dominio lingüístico de cada uno de estos aspectos, para garantizar que la población escolar pueda evolucionar y ser competente en esta área del lenguaje.

\section{Procedimiento metodológico y síntesis de los resultados más relevantes del estudio}

Las sugerencias metodológicas en el aprendizaje de la oralidad en la escuela primaria, las cuales se presentarán a 
continuación, tienen como fundamento los resultados encontrados en una muestra de 36 textos orales narrativos y 36 textos orales explicativos, producidos por treinta y seis estudiantes de primero a sexto grado de una escuela pública de San José. En el caso del texto oral explicativo, este se desarrolló con base en la temática de la independencia de Costa Rica, y en el texto narrativo se utilizó como estímulo lingüístico una película de animales, que posteriormente los niños narraban con sus propias palabras.

El análisis de los textos orales se delimitó al componente léxico, considerando que es el vocabulario la materia prima o el insumo que permite al escolar la construcción de un texto que responda a un contexto comunicativo y que requiere de la integración de los otros componentes para que este sea comprensible y se adecue a las necesidades de la situación comunicativa. Por lo tanto, se valoró la riqueza léxica, mediante el Test de vocabulario para preescolares costarricenses de 3 a 6 años TEVOPREESC, Murillo (en prensa), con el cual se puede conocer la riqueza léxica de los escolares mediante la descripción de la variabilidad de palabras de contenido léxico tales como sustantivos, adjetivos y verbos en producciones orales de 50 enunciados. Por otro lado, en relación con la precisión léxica, se hizo un análisis cualitativo para identificar los casos de precisiones e imprecisiones utilizadas por los escolares.

Los resultados más relevantes del estudio se presentan a continuación. En el caso de los textos orales narrativos, los índices de diversidad léxica obtenidos por los grados escolares fueron los siguientes: $1^{\circ}(0,58), 2^{\circ}(0,57), 3^{\circ}(0,55), 4^{\circ}(0,57), 5^{\circ}$ $(0,57)$ y $6^{\circ}(0,55)$. Por otro lado, en cuanto a los textos orales explicativos, los estudiantes lograron los siguientes índices de diversidad léxica: $1^{\circ}(0,59), 2^{\circ}(0,61), 3^{\circ}(0,64)$, $4^{\circ}(0,59), 5^{\circ}(0,65)$ y $6^{\circ}(0,63)$. De acuerdo con este comportamiento, no se halló una constante que permitiera establecer que la diversidad del léxico se acrecienta según transcurre el niño en su vida escolar en ambos tipos de texto. En este sentido, no se visualiza una evolución de de este componente de acuerdo el grado escolar.

Por otro lado, con respecto a la precisión léxica, en términos generales se visualizaron en todos los escolares ejemplos de imprecisiones léxicas. En este sentido, fue constante el uso de términos imprecisos con respecto a la categoría gramatical de los sustantivos, tales como: "cosa" "algo" "todo" y "lugar", los cuales sobresalieron tanto en los textos orales narrativos como explicativos. Por otro lado, el uso del verbo hacer, como "hacer cosas", provocó serias imprecisiones en las producciones. Asimismo, la utilización de adjetivos genéricos como bonito/feo y malo/bueno en diversos contextos. Aunado a ello, el término "chunche", el cual es cotidiano escuchar en el habla informal del costarricense, se presentó con frecuencia y provocó imprecisión en la comprensión de las ideas de los textos orales.

En respuesta a la débil variedad y precisión léxica que fue sistematizada en la investigación en cuestión, y con la certeza y el convencimiento de que el léxico es el recurso primordial para construir en forma precisa, concreta y amplia los textos, es que se presenta a continuación una serie de sugerencias metodológicas para el aprendizaje de la expresión oral en los niños escolares, las cuales pretenden guiar el trabajo que realiza el docente en el aula para favorecer la adquisición y desarrollo del código oral en general.

\section{Sugerencias metodológicas para el aprendizaje de la expresión oral en la escuela primaria}

El objetivo primordial de las sugerencias metodológicas que se presentan a continuación es brindar una orientación al docente en relación con el aprendizaje y el desarrollo de la expresión oral en los 
escolares de escuela primaria. En este sentido, se hace una propuesta de tres sugerencias sobre cómo incorporar el desarrollo de los elementos lingüístico-textuales del discurso oral, particularmente el componente léxico de forma graduada y sistematizada, considerando los objetivos y contenidos de la expresión oral descritos anteriormente.

\subsection{Determinar las habilidades y conocimientos de la lengua oral que el estudiante deberá dominar para lograr la competencia discursiva}

Esta primera sugerencia metodológica recae en la necesidad de conocer cuáles son las habilidades y conocimientos de la oralidad necesarios para que el aprendiz se desenvuelva en forma competente de acuerdo con el grado escolar.

En este caso, podrían visualizarse dos tipos de dominio: por un lado los elementos instrumentales de la lengua tales como lo fonético, lo morfológico, lo sintáctico y lo léxico; por otro lado, la expresión clara y coherente de ideas, sentimientos, emociones y vivencias. Tales elementos deben profundizarse a lo largo de la escolaridad hasta lograr su dominio.

A continuación de describen los contenidos y habilidades en cada uno de los componentes lingüístico-textuales del discurso oral, los cuales el docente deberá graduar de acuerdo con el grado escolar:

- Fónico: Considerar la correcta pronunciación de las palabras. En este sentido, según el grado escolar del niño, hay que construir secuencias fónicas más complejas. Trabajar los elementos concernientes a la entonación pero diferenciando tipos de enunciados (exclamativos, interrogativos, entre otros). Además, hay que generar actividades en las que los estudiantes sean capaces de marcar el énfasis en una frase y distinguir los ritmos y grupos tonales.
-Morfosintáctico: El niño deberá dominar la utilización de las unidades mínimas (morfemas) según las reglas establecidas para poder formar elementos mayores. El estudiante debe diferenciar las categorías gramaticales que tiene a su disposición tales como calificativos, determinantes, nombres, pronombres, entre otros y sus funciones dentro del texto. Además, desde el punto de vista sintáctico, son importantes las habilidades que permiten ordenar y combinar morfemas y sintagmas en secuencia así como aquellas con las que se construyen enunciados. En este sentido, es importante que los sujetos puedan establecer diferencias entre oraciones simples y compuestas y puedan construirlas correctamente. La conciencia morfosintáctica tiene un papel relevante porque permite crear un mecanismo de autocorrección y de mejoramiento en la construcción de sus enunciados. -Organizacional y discursivo: Este aspecto se refiere a la estructura del discurso, a saber, inicio, progresión informativa y finalización del mismo. El niño deberá utilizar adecuadamente dentro de la organización del texto las marcas de coherencia tales como marcadores discursivos, conectores y marcas de interacción.

-Léxico: El vocabulario debe enseñarse tanto en amplitud como en exactitud. La variedad y riqueza léxica permite al hablante adaptarse mejor a un tema y participar con más certeza y fluidez en la interacción comunicativa. El aprendizaje del vocabulario debe partir de las mismas temáticas de los programas de estudio y de situaciones que sean significativas para los estudiantes. Este nivel podrá ampliarse mediante el uso de campos semánticos, la formación de nuevas palabras por medio de estrategias de derivación, mediante la comprensión 
de significados y del uso pertinente del vocabulario en un contexto adecuado.

$\mathrm{Al}$ respecto Nippold (1998) sugiere trabajar la amplitud, la definición y las relaciones de las palabras dentro de contextos significativos, en este caso utilizado el razonamiento inductivo mediante las analogías, en las cuales se realizan comparaciones entre conceptos y por otro lado, el razonamiento deductivo a través de los silogismos, donde se relacionan dos proposiciones y se establecen inferencias o conclusiones de ambas. Igualmente se rescata en el aula el trabajo didáctico de la metáfora y la símil.

Todos los contenidos gramaticales citados anteriormente, interiorizados por el niño como los saberes necesarios para ser competente en el uso del discurso oral, requerirán de estrategias metodológicas que orienten al estudiante para que puedan utilizarlos de forma adecuada y coherente de acuerdo con la intención comunicativa. A medida de que el individuo se apropie de ese conocimiento y lo utilice acertadamente, las competencias de la oralidad se fortalecerán y el cúmulo de aprendizaje se convierte en un puente para alcanzar el éxito en otras áreas del currículo escolar. Por ello, es necesario insertar en la estructura del discurso las palabras que aprenden los niños de manera que ejerciten las competencias requeridas para iniciar su discurso, darle progresión y poder finalizarlo. Del mismo modo, es necesario un adecuado uso de marcas de coherencia tales como marcadores discursivos, conectores y marcas de interacción como son los gestos.

\subsection{Establecer un programa sistemático y graduado de la expresión oral que se centre en la adquisición de habilidades y conocimientos}

Esta segunda sugerencia metodológica, puntualiza que todo programa sistemático, graduado y continuo en el aprendizaje de la expresión oral requiere de la planificación de objetivos a largo y corto plazo, los cuales no deberán estar centrados en contenidos sino en el desarrollo de habilidades.

En este sentido, los programas de estudio del área de Español reflejan en los distintos grados de enseñanza el desarrollo de contenidos concernientes a la expresión oral; sin embargo, la gran debilidad del programa radica en la falta de claridad respecto de cómo deben trabajarse esos objetivos y contenidos a lo largo de la escolaridad.

Por lo tanto, es necesario puntualizar los contenidos específicos de la expresión oral para cada grado escolar con sus respectivas metas, objetivos y recomendaciones metodológicas.

En este sentido, la diversidad léxica y el conocimiento gramatical deberán graduarse e integrarse en la construcción de textos orales según las diferentes secuencias textuales tales como la argumentación, la explicación, la narración y la descripción.

Por lo tanto, en principio, se trabajará el vocabulario en forma graduada y los contenidos gramaticales y no gramaticales en situaciones espontáneas en las que los alumnos puedan fortalecer el uso de las estrategias discursivas para, posteriormente, enfocarse en modalidades más formales del discurso oral.

Si el estudiante no ha tenido la oportunidad de vivenciar situaciones discursivas orales donde pueda hacer uso de la palabra con la finalidad de construir un texto coherente en una determinada situación o con una intencionalidad comunicativa específica, difícilmente podrá alcanzar un desenvolvimiento adecuado cuando deba preparar y enfrentar géneros discursivos más formales como la conferencia, mesa redonda, una exposición, entre otros.

Por ello, al planificar la actividad de expresión oral, primeramente debe prestarse mayor atención a la finalidad de la enseñanza del léxico, pues es el vocabulario el que permite estructurar el pensamiento, simbolizar relaciones y representar el mundo. 
Sobre esa sistematización y graduación del aprendizaje de la lengua, Nippold (1998) manifiesta con respecto a la comprensión de estructuras complejas - se ha demostrado en niños de 7 y 12 años- que la utilización de conjunciones subordinadas se asocia a la comprensión de relaciones semánticas más precisas y complejas. No obstante, el proceso de precisión y diferenciación del significado de las conjunciones continúa después de los 12 años.

$\mathrm{Al}$ respecto Rojas (2006 a) menciona los elementos que deben considerar los docentes en la enseñanza del léxico:

- Distinguir entre el léxico ocasional y el léxico planificado, así mismo establecer las materias que regularán el léxico planificado, según disponibilidad, frecuencia de uso, grado de procesamiento verbal, entre otros. -No olvidar que el objetivo primordial es el adiestramiento en el uso; por eso se debe preparar tareas orientadas al conocimiento -ejercicios, explicación de reglas, entre otrosy tareas orientadas a la habilidadpráctica que mejorará las gramáticas de desarrollo de los aprendices.

-Se puede presentar el significado directamente (definición) pero mucho mejor provocar su comprensión mediante claves contextuales (frasetexto), extralingüísticas (referencias, gestos y dibujos) o también claves lingüísticas, relaciones semánticas, paráfrasis, entre otros.

-Ha de conocer bien diversas técnicas de enseñanza del léxico relacionadas con la ausencia o presencia del contexto.

-Sin ayuda del contexto corresponde a opciones léxicas (por ejemplo, seleccionar el paralelo semántico o el antónimo). Esta actividad supone cierta superficialidad en el procesamiento lingüístico y ejercita la memorización mecánica.
-Descubrir la unidad léxica necesaria: hiperónimos, distinción fonológica, técnicas de paráfrasis, entre otras.

-El léxico contextualizado: posibilita la referencia; pero siempre limitada por el contexto (p. 23).

Las recomendaciones anteriores permiten determinar que la graduación y sistematización del léxico debe partir del léxico ocasional, para luego inducir al niño con respecto al léxico planificado. En este proceso es indispensable el trabajo del significado de las palabras, tal como señala la autora, mediante diversas estrategias que el educador puede planificar en cada una de las áreas específicas del currículum escolar.

Todos estos ejercicios pueden ser abiertos, que permitan un vocabulario más creativo, o cerrados, donde se trate de recordar o identificar determinadas formas léxicas.

De esta manera, la enseñanza planificada del componente léxico permite al docente propiciar en los estudiantes el uso del vocabulario adquirido en la construcción de textos orales.

Asimismo, los textos orales deberán adecuarse al grado escolar de cada alumno: los niños de primer ciclo podrían optar por los textos descriptivos, narrativos y explicativos; para el segundo ciclo se recomienda considerar el nivel de complejidad los textos orales argumentativos. Los siguientes lineamientos metodológicos podrían facilitar dicha sistematización para gestar el proceso de adquisición y desarrollo del código oral:

-En primer y segundo grado se sugiere trabajar el vocabulario a partir de las mismas temáticas que se desarrollan en clase y contextualizarlo en géneros discursivos tales como cuentos, fábulas, descripciones, rimas, entre otros, con el propósito de trabajar la diversidad y precisión del vocabulario en la construcción de tipologías textuales y proyectar esta competencia en la adquisición del plano semántico, fónico, sintáctico y 
morfológico y pragmático. Todo ello adecuadamente dosificado.

-En tercer grado se recomienda continuar con el trabajo sugerido para primer y segundo grado y extenderlo con el desarrollo de la lectura oral de diferentes tipos de texto, considerando aspectos como las pautas, la entonación, el ritmo, entre otros.

Además, a partir de esa lectura oral, ya sea de noticias, historias y otros textos, se podrá fomentar en los estudiantes la reconstrucción y producción de producciones orales.

Se recomienda impulsar la discusión, criticidad y defensa de ideas sobre una temática, así como realizar investigaciones y exponer resultados, esto con el propósito de fomentar la argumentación en la población escolar. En este sentido, la secuencia didáctica como una alternativa en la adquisición y desarrollo de las competencias orales de los escolares permite establecer pautas claras acerca de la construcción del discurso, ya que es una estrategia que permite establecer primeramente una planificación de lo que se aprenderá y cómo se hará, así como las herramientas y conocimientos necesarios para producir el discurso, y por último un espacio de evaluación y autorreflexión, que permita determinar los logros obtenidos en el proceso de aprendizaje.

- En cuarto grado se sugiere articular el trabajo realizado en el primer ciclo y ampliarlo con espacios que permitan al estudiante analizar su propia expresión oral. En este caso, se puede hacer uso de recursos audiovisuales como la grabadora, las cámaras de video y listas de cotejo, en el caso de estas últimas, los parámetros que se incluyan deberán ser modelados, ejercitados y discutidos de antemano en clase.

-En quinto y sexto se recomienda articular el trabajo valorativo de la expresión oral forjado en el primer ciclo y el cuarto grado, para retroalimentarlo y afinarlo aún más con respecto al nivel organizacional $\mathrm{y}$ discursivo, con el objetivo de que los estudiantes mejoren en la coherencia y cohesión de sus ideas. Para ello se deben habilitar espacios en donde los niños participen de exposiciones, foros, debates y mesas redondas, lo que a su vez generará en los escolares el desarrollo de la comprensión, el razonamiento y la interpretación.

Por otra parte, el docente debe cuidar ciertas actitudes que pueden entorpecer el aprendizaje de la oralidad. Domínguez, citado por Martín (2009), hace referencia a dichas actitudes que deben practicarse con el estudiante:

- Hablarle sin provocar una respuesta estándar.

- Repetirle correctamente el mensaje sin corregirle.

- No llamarle la atención en grupo sobre su falta o trastorno.

- Formar un clima de confianza con el que se refuercen las adquisiciones y logros de los niños, también en relación con determinados tipos de trastornos (como el tartamudeo). Igualmente, se recomienda no alabarlos cuando acierten porque podría recordarles su trastorno.

- Proporcionarle situaciones de diálogo, aprendizaje de canciones y situaciones lúdicas en las que hable y pregunte. Hay que prestar atención al contenido y brindar modelos correctos.

- Buscar un estímulo interesante y realizar preguntas abiertas ya que el estudiante sabe cuándo el docente conoce la respuesta. No hay que dirigir ni intervenir demasiado en el proceso para que sean los alumnos quienes dirijan la conversación.

- No alargar las preguntas ya que provoca respuestas cortas; hay que 
diversificar los temas de conversación y respetar los turnos de habla.

En general, la valoración de la expresión oral debe visualizarse como un proceso más que un producto; por lo tanto, la sistematización de habilidades y conocimientos por nivel escolar es transcendental para establecer una adecuada articulación entre habilidades y conocimientos en relación con la adquisición del código oral en la escuela costarricense.

\subsection{Establecer criterios para valorar o evaluar la expresión oral de los estudiantes}

La última sugerencia metodológica establece que una vez identificados los tipos de textos orales que se trabajarán con sus respectivos conocimientos gramaticales y habilidades, es necesario que el docente se plantee cuáles serán las estrategias que se utilizarán para la corrección, es decir, cómo se realizará la evaluación o valoración.

En este sentido, lo fundamental en la adquisición y desarrollo de las competencias es el proceso y no el producto; de ahí la importancia de la evaluación formativa del discurso oral, la cual no gira en torno a un resultado final, sino más bien a un proceso de aprendizaje que pretende vincular habilidades y estrategias que posibiliten la planificación del discurso oral formal.

Dentro de la evaluación formativa Camps, citada por Vilá et ál. (2005), plantea el modelo de secuencia didáctica en la planificación del discurso:

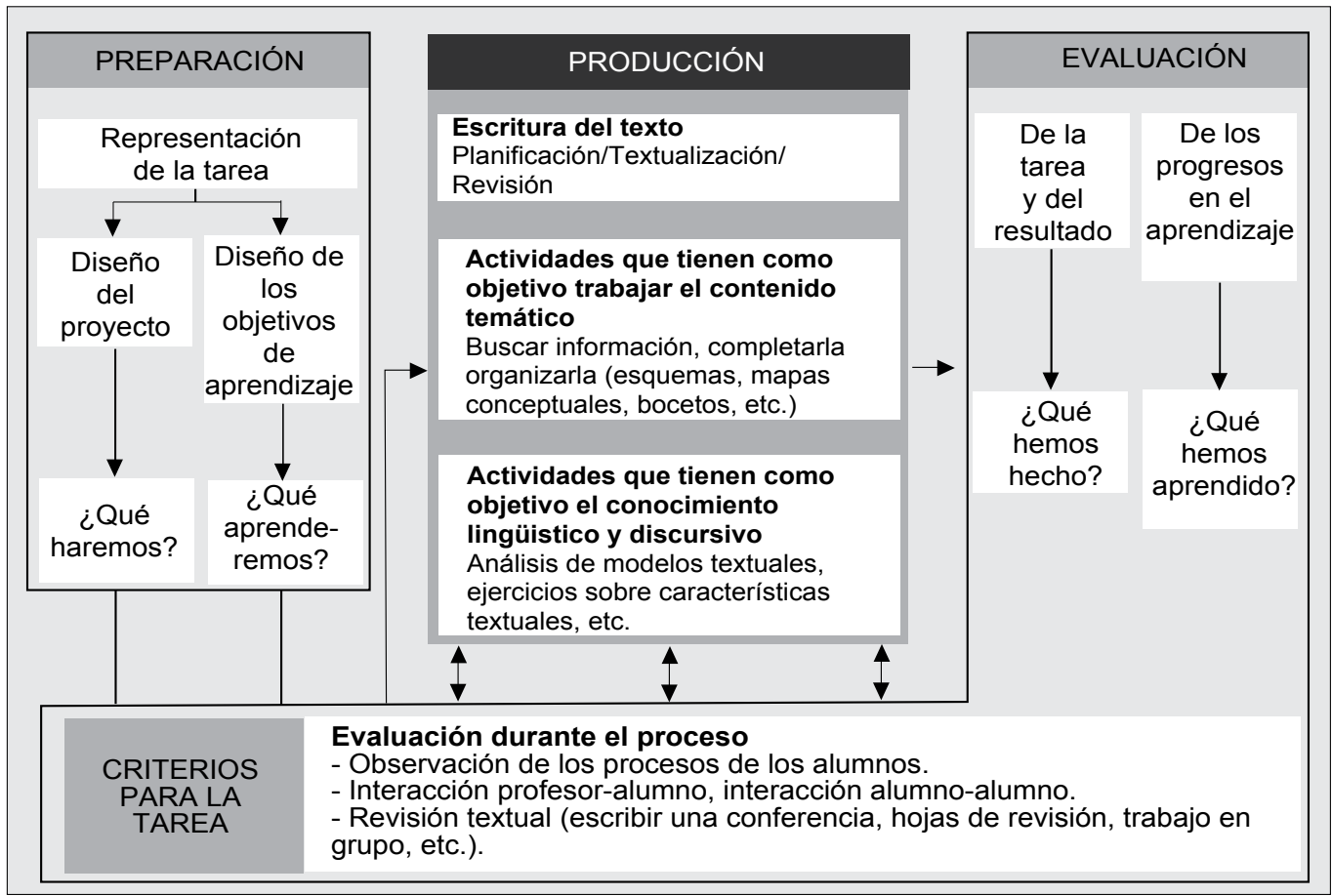

Figura $\mathrm{N}^{\circ} 1$ : Modelo de secuencia didáctica

Fuente: Camps, citado por Vilá et ál. (2005: 122) 
Tal como se aprecia en el gráfico anterior, la secuencia didáctica desarrolla tres etapas básicas: la preparación, la cual permite la planificación de la tarea y de lo que se espera lograr; luego, la producción permite al sujeto el desarrollo de la propuesta planificada y por último la evaluación de lo logrado. La secuencia didáctica, como una alternativa en la adquisición y desarrollo de las competencias orales de los escolares, induce al establecimiento de pautas claras acerca de la construcción del discurso. Además, posibilita al docente graduar los objetivos y contenidos de la oralidad de una forma sistemática y rigurosa y, a la vez, fortalecer las competencias de la misma.

Cuando se establece este tipo de estrategias se enfatiza el proceso de la composición del discurso oral, puesto que se busca que los estudiantes sigan pautas para poner en práctica las estrategias lingüísticas y discursivas y generen espacios tanto para la replanificación del texto como para la profundización de los temas.

En este sentido, con la finalidad de que el escolar produzca textos que cumplan con el propósito planteado, el componente léxico debe renovarse constantemente de acuerdo con las experiencias del niño y el nuevo conocimiento que adquiere, así como orientarse y aprovecharse en la producción tanto oral y escrita de diversos textos.

Este alcance se puede concretar mediante la propuesta de vocabulario para trabajar en primer y segundo ciclos de la Educación General Básica elaborada por Murillo (sin publicar), en la que se describe una metodología en la que se activan dos estrategias básicas para la enseñanza del vocabulario: la instrucción directa y la indirecta. La primera comprende los siguientes elementos:

-El desarrollo de la competencia definicional en el niño: Se refiere al desarrollo de la aptitud para transmitir el significado de las palabras de manera pertinente de acuerdo con su uso y en los contextos adecuados.
-Uso del diccionario: Su uso debe iniciarse a partir del primer ciclo, con el propósito de esclarecer el significado y el uso de las palabras.

-Análisis morfológico: Debe desarrollarse a partir de cuarto grado. Permite la comprensión de las palabras y sus partes con el objetivo de aprender nuevas palabras.

-Establecimiento de relaciones y asociaciones léxico-semánticas: Se vincula el desarrollo del vocabulario a partir de las relaciones semánticas más representativas, tales como sinónimos, antónimos, inclusión de clases y el estudio de campos semánticos.

Por otro lado, la instrucción indirecta apuesta por el desarrollo de las siguientes estrategias:

-La lectura extensiva e independiente: Tiene como objetivo estimular la lectura para el desarrollo del vocabulario y la comprensión de los textos. -La lectura intensiva: Tiene como propósito que el alumno no solo comprenda el texto, sino que identifique el vocabulario, los elementos gramaticales, la tipología textual, los argumentos, entre otros.

-Las claves contextuales: Estas se accionan cuando el contexto comunicativo determina el significado de la palabra, en principio desconocido para el estudiante.

Se recomienda que tanto la instrucción directa y la indirecta se trabajen en forma complementaria, dependiendo de las estrategias y objetivos planteados con antelación por el docente.

De esta manera, el esbozo de las pautas didácticas para el aprendizaje de la expresión oral sugerido en este artículo, pretende que ellas sean guías, lineamientos $\mathrm{u}$ orientaciones para que los docentes proyecten la enseñanza del vocabulario 
mediante un sistema graduado y sistematizado de habilidades y destrezas.

\section{Conclusiones}

El establecer por cada grado escolar los conocimientos lingüísticos y no lingüísticos en la adquisición y el desarrollo de la competencia comunicativa oral repercute de manera positiva en el progreso y dominio de esta habilidad. Por ello, los centros escolares tienen una función primordial en la sistematización y graduación de este saber, el cual el estudiante deberá adquirir a lo largo de la escolaridad para convertirse en un hablante competente.

En cuanto a la competencia léxica, es importante que el docente incorpore dentro de su mediación pedagógica el aprendizaje del vocabulario, ya que es el insumo elemental para que los estudiantes estructuren y amplíen sus producciones orales y escritas.

En el sistema educativo escolar costarricense, no es posible que la adquisición y el desarrollo del discurso oral se sustenten de improvisaciones, supuestos e inclusive se margine en comparación con la importancia del aprendizaje de la lectura y la escritura. En este sentido, se deben desplazar criterios únicos que han direccionado la adquisición y evaluación de la expresión oral, tales como la corrección de palabras por una deficiente pronunciación y la implementación inadecuada del plano morfológico.

Es trascendental el fortalecimiento de la adquisición de la lengua oral con la incorporación de los aspectos sintácticos y semánticos, así como el componente organizacional discursivo, con el objetivo de desarrollar producciones de textos orales coherentes y con la presencia de vocabulario diverso y preciso, además de ser vinculantes con todas áreas del currículo escolar, y no únicamente en la asignatura de Español.
La escasa o nula evaluación del componente léxico en los escolares presente en la investigación que fundamenta este artículo puede ser enriquecida por medio de pautas metodológicas que integren no solo conocimientos lingüísticos sino también habilidades, las cuales deben ser trabajadas en forma gradual en los diferentes niveles escolares.

La evaluación de la expresión oral requiere de un planteamiento o modelo de evaluación distinto al que en ocasiones se percibe en el aula costarricense, el cual se reduce a las exposiciones memorísticas de textos aislados de la propia realidad del niño. En este sentido, si lo que se pretende es un escolar competente en el área oral, se debe iniciar por la práctica constante de la conversación en el aula; sólo de esa forma se potencializa el verdadero sentido comunicativo del uso de la palabra, para posteriormente orientar las habilidades comunicativas a géneros más formales como las conferencias, mesas redondas, debates, entre otros.

\section{Referencias bibliográficas}

Araya, J. (2011) La competencia en la expresión oral de niños escolares en Costa Rica. El componente léxico. (Tesis de posgrado). Universidad de Costa Rica.

Azagra et ál. (1998). Lev Vigotsky: sus aportes para el siglo XXI. Cuadernos de Publicación UCAB: Caracas

Barboza , G y Delgado, S. (2008). La expresión escrita en la escuela primaria. (Tesis de grado).Universidad de Costa Rica.

Calsamiglia, H. y Tusón, A. (2008). Las cosas del decir. Barcelona: Ariel.

Cassany, D. et al. (1994). Enseñar lengua. Bacelona: Graó

Lomas, C.(1999) Cómo enseñar a hacer cosas con palabras. (Vol 1). Barcelona: Paidós. 
Martín, R. (2009). Manual de Didáctica de la Lengua y Literatura. Madrid:Síntesis.

Ministerio de Educación Pública (2004). Programa de Estudio de Español. San José: Departamento de publicaciones, Ministerio de Educación Pública.

Ministerio de Educación Pública. (1994). Política Educativa Siglo XXI. San José: Departamento de publicaciones, Ministerio de Educación Pública.

Murillo, M (1999). Crecimiento de la disponibilidad léxica: niños de preescolar y primer ciclo de la educación básica costarricense. Revista de Filología y Lingüistica de la Universidad de Costa Rica, 25(2), 187-203.

Murillo, M. (2003). Estudio sobre el lenguaje de los escolares costarricenses: El léxico básico y la ortografía y sus características.(Tesis doctoral),Universidad de Extremadura, España.

Murillo, M. (2008). La educación lingüística en la educación primaria costarricense. Káñina, Revista de Artes y Letras de la Universidad de Costa Rica, 23(2), 69-91.

Murillo, M. (sin publicar). Propuesta de vocabulario para trabajar en I y II ciclos de la Educación General Básica. La palabra como objeto de enseñanza y aprendizaje. Informe final de investigación de la Universidad de Costa Rica.

Murillo R, M y Sánchez,V. (2002). Crecimiento del léxico básico de los escolares costarricenses: código oral. Káninina, Revista de Artes y Letras de la Universidad de Costa Rica, 24(2), 97-108.
Murillo R, M. y Sánchez, V. (2004). Diaintegración, contrastividad y vocabulario de los escolares costarricenses. Káninina, Revista de Artes y Letras de la Universidad de Costa Rica, 28(2), 133-146.

Murillo, M. y Sánchez, V. (2005). Elementos diaintegrativos del léxico básico escolar costarricense. Káñina, Revista de Artes y Letras de laUniversidad de Costa Rica,. 29 (edición especial), 165-177.

Nippold. M. (1998). Later language Development. Texas: Pro-ed. An International Publisher.

Palou, J.et ál. (2005). La lengua oral en la escuela. Barcelona: Graó.

Reyzábal, V. (1993). La comunicación oral y su didáctica. Madrid: La Muralla.

Rojas, M. (2002). Léxico básico escolar costarricense. Informe de Investigación. Universidad de Costa Rica.

Rojas, M (2005). Una responsabilidad escolar olvidada: el desarrollo del componente léxico. Revista Educación de la Universidad de Costa Rica.

Rojas, M. (2006a). El léxico meta para la escuela costarricense. Revista Actualidades Investigativas en Educación, 6(3), 1-26.

Rojas, M. (2006b). Léxico meta para la escuela costarricense: esbozo de una propuesta metodológica para el desarrollo de la madurez léxica.(Tesis de posgrado).Universidad de Costa Rica.

Rojas, M. et ál. (2008). Palabras juguetonas. Dinámicas con léxico escolar gradual. San José: SIEDIN-UCR

Vilá, M. et ál. (2005). El discurso oral formal. Barcelona: Graó. 
